\title{
Ultrasonography for Detecting Adhesions: Aspirin Continuation for Lung Resection Patients
}

\author{
MOTOAKI YASUKAWA ${ }^{1}$, RYOSUKE TAIJI ${ }^{2}$, NAGAAKI MARUGAMI ${ }^{2}$, TAKESHI KAWAGUCHI $^{1}$, \\ NORIKAZU KAWAI ${ }^{1}$, NORIYOSHI SAWABATA ${ }^{1}$, TAKASHI TOJO ${ }^{3}$, \\ JUNKO TAKAHAMA ${ }^{2}$, NAOKI HAMAZAKI ${ }^{4}$, TOSHIKO HIRAI ${ }^{5}$ and SHIGEKI TANIGUCHI ${ }^{1}$ \\ ${ }^{1}$ Department of Thoracic and Cardiovascular Surgery, Nara Medical University School of Medicine, Nara, Japan; \\ ${ }^{2}$ Department of Radiology, and ${ }^{3}$ Department of Thoracic Surgery, Saiseikai Chuwa Hospital, Nara, Japan; \\ ${ }^{4}$ Shioya Clinic of Internal Medicine, Nara, Japan; \\ ${ }^{5}$ Department of Endoscopy and Ultrasound, Nara Medical University School of Medicine, Nara, Japan
}

\begin{abstract}
Background/Aim: Aspirin reduces cardiovascular disease and/or stroke risks. However, perioperative aspirin use remains controversial. We assessed the efficacy of ultrasonography to facilitate video-assisted thoracic surgery (VATS). We analyzed the perioperative management of patients using aspirin and its association with bleeding events during lung cancer surgery. Patients and Methods: A total of 38 patients who underwent VATS after continuing or discontinuing aspirin were examined. Ultrasound was performed preoperatively to evaluate the pleural adhesions. Fisher's exact test was used to analyze correlations between the two groups. Results: Dense adhesions were found at VATS ports using ultrasonography (accuracy: 100\%). No differences were detected in bleeding, thrombotic events, or operative times between the aspirin and non-aspirin groups. There were differences in bleeding $(p=0.009)$ and operative times $(p=0.021)$ between the dense adhesion and non-dense adhesion groups. Conclusion: Preoperative detection of pleural adhesions using ultrasonography was useful in selecting pulmonary resection patients who continued aspirin perioperatively.
\end{abstract}

During the past two decades, video-assisted thoracic surgery (VATS) has been established as the preferred approach for almost all thoracic surgical procedures. Recently, several authors have reported the efficacy of uniportal VATS for

This article is freely accessible online.

Correspondence to: Motoaki Yasukawa, Department of Thoracic and Cardiovascular Surgery, Nara Medical University School of Medicine, Kashihara, Nara 634-8522, Japan. Tel: +81 744223051, Fax: +81 744248040, e-mail: myasukawa@naramed-u.ac.jp

Key Words: Pleural adhesion, ultrasonography, aspirin. lobectomy $(1,2)$. However, there is no agreement regarding the limitations of this method. The most popular technical contraindications for VATS for pulmonary resection are dense pleural adhesions, incomplete interlobar fissures, previous chemotherapy and/or radiotherapy, perivascular and/or peribronchial fibrosis, tumors larger than $5 \mathrm{~cm}$, chest wall involvement, centrally located tumor, severe comorbidity, advanced age, severe chronic obstructive pulmonary disease (COPD), and emphysema (3). For experienced surgeons, VATS in the presence of dense pleural adhesions is controversial. Furthermore, for inexperienced surgeons, VATS in the presence of dense pleural adhesions could lead to unexpected massive bleeding and/or lung injury (4). Preoperative transthoracic ultrasonography is useful for detecting pleural adhesions in patients scheduled to undergo thoracotomy (5-7). We speculated that preoperative evaluation of pleural adhesions is beneficial before surgical intervention.

In Japan, many elderly patients with lung cancer undergo pulmonary resection. However, they often have comorbidities, including cardiovascular and/or cerebrovascular diseases (8). Because aspirin has been used for decades as secondary prevention of myocardial infarction or stroke by patients with ischemic heart or cerebrovascular disease (9), increasing numbers of lung cancer patients with cardiovascular and/or cerebrovascular diseases are using aspirin. Major adverse cardiac and cerebrovascular events (MACCEs) are common causes of disabling and/or fatal outcomes after non-cardiac surgery $(10,11)$. Therefore, there is a clinical dilemma regarding patients who use aspirin before surgery in terms of whether aspirin treatment should be continued because of the risks of thromboembolic complications compared with the risks of coagulopathy. Recent data regarding the risk of discontinuing antiplatelet therapy by patients with coronary stents have highlighted the use of aspirin during the perioperative period $(12,13)$. Therefore, the routine withdrawal of aspirin 7-10 days before surgery has been 
questioned, and some recent publications have recommended that aspirin should not be stopped during the perioperative period $(14,15)$. However, these recommendations were not based on evidence from controlled trials that could elucidate the risks and benefits of aspirin in the setting of non-cardiac surgery.

Kanzaki et al. (4) demonstrated the feasibility of aspirin continuation during the perioperative period for lung cancer patients requiring pulmonary resection. However, they reported that 1 out of 20 patients who continued using aspirin had blood loss of $500 \mathrm{~g}$ intraoperatively and postoperative bleeding that required transfusion due to an intrathoracic adhesion. Considering their report, it is important to determine whether there are thoracic adhesions in patients who continue using aspirin. This study aimed to assess the efficacy of ultrasonography before VATS to analyze the efficacy of perioperative management with antiplatelet therapy, especially aspirin, for such patients and the relationship between aspirin and VATS regarding the incidence of MACCE and major bleeding events during lung cancer surgery.

\section{Patients and Methods}

Patients. Among 287 patients who underwent lung cancer resection at the Nara Medical University Hospital from January 2017 to December 2018, 38 who underwent lung cancer resection and were using aspirin were enrolled. The exclusion criterion for entry into the study population was the use of other anticoagulation drugs and/or antiplatelet drugs. All 38 patients underwent preoperative ultrasonography to evaluate the possible presence of pleural adhesions.

Of 38 patients who underwent lung cancer resection from January 2017 to September 2017, 10 stopped using aspirin 7 days before surgical intervention (group 1). The other 28 patients who underwent lung cancer resection from October 2017 to December 2018 had continued using aspirin before surgical intervention and did not have adhesions according to ultrasonography (group 2).

Patients and methods. We used VATS to resect the lung tumors. This involved four incisions: an incision along the anterior axillary line for a camera port (initial port) in the seventh or eighth intercostal space; a 1-cm incision along the anterior axillary line in the fourth or fifth intercostal space; a 1-cm incision along the posterior axillary line in the seventh or eighth intercostal space; and a 4- to 5-cm incision in the axilla for a working port in the fourth or fifth intercostal space.

The ultrasound study was performed within 2 weeks before the scheduled surgery. The LOGIQ E9TM (GE Healthcare, Chicago, IL, USA) ultrasound system was used. All patients were examined in the lateral position with the fourth or fifth intercostal spaces and the seventh or eighth intercostal spaces of the mid-axillary line undergoing respiration. We evaluated the lung sliding sign, which is the movement of visceral pleural sliding with breathing and is a hallmark of the absence of pleural adhesions. If pleural adhesions were detected during preoperative ultrasonography, then patients in group 2 stopped using aspirin 7 days before surgery. If there were no pleural adhesions during preoperative ultrasonography, then patients in group 2 continued using aspirin. In group 2, no patient stopped using aspirin 7 days before surgery.

We checked whether there were adhesions and determined their locations during VATS. Loose adhesions and dense adhesions were considered blunt ablations that could or could not be removed easily. Prolonged air leakage was defined as an air leak lasting for more than 5 days postoperatively. The chest tube duration was measured in days.

The Ethics Review Board of our institute approved the study protocol and waived the requirement to obtain written informed consent from patients because individual patients were not identified in this retrospective study.

Statistical analyses. Either the Chi-square test or Fisher's exact test was performed, as appropriate, to analyze correlations between the two groups. All $p$-values were two-sided, and $p \leq 0.05$ was considered statistically significantly different. All analyses were conducted using EZR on R commander version 1.33 (Saitama, Japan) (16).

\section{Results}

No pleural adhesions were found using preoperative ultrasonography for patients in group 2; these patients were instructed to stop using aspirin before surgery. Table I shows the clinical characteristics of all patients. Ten adhesions were found at VATS ports. Adhesions could not be detected in nine patients. For these nine patients, we were able to remove these adhesions easily, without lung injury and massive bleeding, because they were loose. Visceral slide sonography detected the following adhesions: 1 true-positive adhesion; 9 false-negative adhesions; 0 false-positive adhesions; and 28 true-negative adhesions (sensitivity: 10.0\% [1/10]; specificity: $100.0 \%$ [28/28]; accuracy: $76.3 \%$ [29/38]) (Table II). The lung sliding sign could distinguish between dense adhesions and non-dense adhesions (accuracy: 100\% [38/38]) (Table III). At the initial port, we could not detect two adhesions (accuracy: 94.7\% [36/38]) (Table IV). However, there were 19 adhesions at other sites, including the intrathoracic space and additional port sites. Adhesions were not estimated using ultrasonography at areas other than port sites in this group.

Table I shows the outcomes of surgical intervention. Surgery times for group 1 and group 2 were $109 \pm 47 \mathrm{~min}$ and $116 \pm 53 \mathrm{~min}$, respectively $(p=0.702)$. Intraoperative bleeding in group 1 and group 2 comprised $21.2 \pm 24.2 \mathrm{~g}$ and $23.3 \pm 39.0$ $\mathrm{g}$, respectively $(p=0.877)$. No patients underwent blood transfusion intraoperatively or perioperatively. The chest tube durations for group 1 and group 2 were $1.5 \pm 0.8$ days and $3.1 \pm 4.1$ days, respectively $(p=0.235)$. Fortunately, there were no complications, such as postoperative bleeding, myocardial infarction, cerebral infarction, arrhythmia, and pneumonia, among all patients. Three patients in group 2 experienced prolonged air leakage (more than 5 days). Furthermore, we analyzed the outcomes of dense adhesions and of loose or no adhesions. Table $\mathrm{V}$ shows the outcomes of surgical 
Table I. Clinical characteristics of all patients $(n=38)$.

\begin{tabular}{|c|c|c|c|c|}
\hline & All patients, $\mathrm{n}=38(\%)$ & Group $1, \mathrm{n}=10(\%)$ & Group 2, n=28 (\%) & $p$-Value \\
\hline Age (years) & $72.5 \pm 6.5$ & $71.1 \pm 8.2$ & $73.0 \pm 5.9$ & 0.445 \\
\hline Gender & & & & 1.000 \\
\hline Male & $30(78.9)$ & $8(80.0)$ & $22(78.6)$ & \\
\hline Female & $8(21.1)$ & $2(20.0)$ & $6(21.4)$ & \\
\hline Indication for aspirin & & & & 0.890 \\
\hline CAD & 26 & 8 & 18 & \\
\hline Cerebral infarction & 13 & 3 & 10 & \\
\hline Peripheral vascular disease & 2 & 0 & 2 & \\
\hline Others & 1 & 0 & 1 & \\
\hline Procedure & & & & 0.888 \\
\hline Lobectomy & $19(50.0)$ & $6(60.0)$ & $13(46.4)$ & \\
\hline Segmentectomy & $2(5.3)$ & $0(0.0)$ & $2(7.2)$ & \\
\hline Wedge resection & $17(44.7)$ & $4(40.0)$ & $13(46.4)$ & \\
\hline Thoracic adhesions observed & & & & 0.601 \\
\hline Dense & $15(39.5)$ & $3(30.0)$ & $12(42.9)$ & \\
\hline Loose & $5(13.2)$ & $2(20.0)$ & $3(10.7)$ & \\
\hline None & $18(47.4)$ & $5(50.0)$ & $13(46.4)$ & \\
\hline Adhesions sites & & & & NS \\
\hline Ports & 10 & 2 & 8 & \\
\hline Others & 19 & 5 & 14 & \\
\hline Length of surgery (min) & $114 \pm 51$ & $109 \pm 47$ & $116 \pm 53$ & 0.702 \\
\hline Intraoperative bleeding $(\mathrm{g})$ & $22.7 \pm 35.3$ & $21.2 \pm 24.2$ & $23.3 \pm 39.0$ & 0.877 \\
\hline Chest tube duration (days) & $2.7 \pm 3.6$ & $1.5 \pm 0.8$ & $3.1 \pm 4.1$ & 0.235 \\
\hline \multirow[t]{2}{*}{ Postoperative complications } & $3(7.9)$ & $0(0.0)$ & $3(10.7)$ & 0.552 \\
\hline & & & (prolonged air leakage: $>5$ days) & \\
\hline
\end{tabular}

CAD: Coronary artery disease; NS: non-significant.

intervention. Operative times for the dense adhesions group and for the loose or no adhesions group were $143 \pm 53 \mathrm{~min}$ and $97 \pm 46 \mathrm{~min}$, respectively $(p=0.021)$. Intraoperative bleeding for the dense adhesions group and for the loose or no adhesions group comprised $44.7 \pm 52.4 \mathrm{~g}$ and $7.2 \pm 8.8 \mathrm{~g}$, respectively $(p=0.009)$. No patients underwent blood transfusion intraoperatively or perioperatively. Chest tube durations for the dense adhesions group and for the loose or no adhesions group were 5.0 \pm 5.8 days and 1.7 1.2 days, respectively $(p=0.033)$. Three patients had prolonged air leakage; these three were in the dense adhesions group.

\section{Discussion}

We demonstrated that preoperative detection of pleural adhesions using transthoracic ultrasonography could provide safe thoracoscopic access without unexpected massive bleeding and/or lung injury and facilitate VATS among lung cancer patients who continue using aspirin during the perioperative period.

Aspirin (acetylsalicylic acid) is part of the salicylate family of drugs. Universally, it is used as an analgesic, antipyretic, and anti-inflammatory agent. However, primarily because of its antiplatelet effects, it is commonly used for secondary prevention of cardiovascular and neurovascular morbidity of patients with known ischemic heart disease and cerebrovascular disease (i.e., previous myocardial infarction, coronary artery disease, peripheral vascular disease, coronary stent implantation, previous cerebral vascular accident). Aspirin decreases the risk of cardiovascular disease and/or stroke through its antiplatelet characteristics in those at risk for thrombotic events. Within the perioperative period, aspirin use remains controversial. If withheld before surgery, then patients are at risk for cardiac and neurovascular events during the aspirin-free period. Several studies of major surgery (cardiac and non-cardiac) found that aspirin use was associated with increased bleeding; however, others showed minimal bleeding risks and improved outcomes (17-20).

Which patients should continue using aspirin during the perioperative period? The American College of Chest Physicians (ACCP) guidelines in 2012 (21) provided grade $1 \mathrm{~A}$ recommendations for patients with established coronary artery disease, suggesting that long-term single antiplatelet therapy with aspirin is appropriate.

Oscarsson et al. (18) performed a randomized, doubleblinded, placebo-controlled trial to evaluate the effects of aspirin on major adverse cardiac events (MACEs) and found no increase in perioperative bleeding between the aspirin and 
Table II. Results after the lung slide sign were discovered using ultrasonography to detect pleural adhesions. All ports among all patients $(n=38)$.

\begin{tabular}{lcc}
\hline Adhesions predicted & \multicolumn{2}{c}{ Adhesions observed } \\
\cline { 2 - 3 } & Yes & No \\
\hline Yes & 1 & 0 \\
No & 9 & 28 \\
\hline
\end{tabular}

Table III. Results after the lung slide sign were discovered using ultrasonography to detect pleural adhesions at port sites. Adhesion levels among all patients: dense adhesions vs. loose or no adhesions $(n=38)$.

\begin{tabular}{lcc}
\hline Adhesions predicted & \multicolumn{2}{c}{ Dense adhesions observed } \\
\cline { 2 - 3 } & Yes & No \\
\hline Yes & 1 & 0 \\
No & 0 & 37 \\
\hline
\end{tabular}

Table IV. Results after the lung slide sign were discovered using ultrasonography to detect pleural adhesions. Adhesions in the initial port among all patients $(n=38)$.

\begin{tabular}{lcc}
\hline Adhesions predicted & \multicolumn{2}{c}{ Adhesions observed } \\
\cline { 2 - 3 } & Yes & No \\
\hline Yes & 0 & 0 \\
No & 2 & 36 \\
\hline
\end{tabular}

non-aspirin groups. Mantz et al. (19) reported that no difference was detected in bleeding or thrombotic events between the aspirin and non-aspirin groups. The European Society of Cardiology (22) stated that continuation of aspirin by patients previously treated with aspirin should be considered during the perioperative period, but that discontinuation of aspirin therapy should be considered only for those in whom hemostasis is expected to be difficult to achieve during surgery.

We recognize that lung cancer surgery should be performed safely with less bleeding. However, resection of the pulmonary parenchyma may lead to unexpected bleeding due to dense adhesions. Kanzaki et al. (4) demonstrated the feasibility of aspirin continuation during the perioperative period for lung cancer patients requiring pulmonary resection. Furthermore, the need to continue aspirin therapy during the perioperative period should be evaluated on an individual basis. They reported that 1 out of 20 patients who
Table V. Clinical characteristics of patients who continued aspirin $(n=28)$.

\begin{tabular}{lccc}
\hline & $\begin{array}{c}\text { Loose or no } \\
\text { adhesions } \\
(\mathrm{n}=16)(\%)\end{array}$ & $\begin{array}{c}\text { Dense } \\
\text { adhesions } \\
(\mathrm{n}=12)(\%)\end{array}$ & $p$-Value \\
\hline Age (years) & $71.7 \pm 5.7$ & $74.7 \pm 6.0$ & 0.193 \\
Gender & $11(68.8)$ & $11(91.7)$ & 0.196 \\
Male & $5(31.2)$ & $1(8.3)$ & \\
Female & & & 0.769 \\
Procedure & $6(37.5)$ & $7(58.3)$ & \\
Lobectomy & $2(12.5)$ & $0(0.0)$ & \\
Segmentectomy & $8(50.0)$ & $5(41.7)$ & $0.021^{*}$ \\
Wedge resection & $97 \pm 46$ & $143 \pm 53$ & $0.009^{*}$ \\
Length of surgery (min) & $7.2 \pm 8.8$ & $44.7 \pm 52.4$ & $0.033^{*}$ \\
Intraoperative bleeding (g) & $1.7 \pm 1.2$ & $5.0 \pm 5.8$ & 0.067 \\
Chest tube duration (days) & $0(0.0)$ & $3(25.0)$ & 0.067 \\
Postoperative complications & \multicolumn{3}{c}{ (prolonged air } \\
& \multicolumn{3}{c}{$>5$ days) } \\
& \multicolumn{3}{c}{} \\
\hline
\end{tabular}

*Statistically significant.

continued using aspirin experienced intraoperative blood loss of $500 \mathrm{~g}$ and postoperative bleeding on postoperative day 1 that required transfusion due to an intrathoracic adhesion. Considering their report, it is important to determine whether patients who continue using aspirin have thoracic adhesions. Furthermore, it is important to preoperatively select appropriate lung cancer patients who would be at low risk for bleeding. Therefore, we evaluated thoracic adhesions using ultrasonography to select the appropriate patients. If patients are at high risk for bleeding, then they should not continue using aspirin because the risk of perioperative bleeding complications might be increased. However, if they are not at high risk for bleeding, then they should continue aspirin because the risk of perioperative thromboembolic complications might be increased due to discontinuing the use of aspirin.

Preoperative transthoracic ultrasonography was useful for detecting pleural adhesions in patients who have undergone thoracic surgery (5-7). When we could not detect adhesions in patients who, in fact, did have adhesions, the adhesions were not dense and could be removed easily without injuring the lung. Therefore, we used preoperative ultrasonography to evaluate the feasibility of aspirin continuation during the perioperative period before pulmonary resection to provide safe thoracoscopic access, without lung injury and/or unexpected massive bleeding, thereby facilitating VATS. The lung sliding sign can distinguish between dense adhesions and non-dense adhesions. If there were dense adhesions, then the lung would not slide with breathing. If 
there were no adhesions or if there were loose adhesions, then the lung would slide with breathing. Regarding the safety of VATS, especially when there are adhesions, it is important to not distinguish between adhesions and no adhesions; instead, one should distinguish between easy cases and difficult cases and between high potential and low potential for complications.

In this study, no differences were detected in bleeding events, thrombotic events, and operative time between the aspirin group (group 2) and non-aspirin group (group 1). However, there were differences in bleeding and operative times between the dense adhesions group and the non-dense or no adhesion group. Therefore, using ultrasonography might be helpful when selecting appropriate lung cancer patients continuing aspirin who could experience fewer complications during VATS. Furthermore, the presence of adhesions might be a prognostic factor for prolonged postoperative air leakage.

Here, we used preoperative ultrasonography to determine the existence of adhesions in lung cancer patients and the severity of their coexisting cardiovascular or cerebrovascular diseases, type of stent implanted, and duration of stent use. Unfortunately, we did not research the expected port site adhesions. Detecting adhesions near the apex area when using ultrasonography is difficult. If we are careful when removing adhesions during VATS, then we can perform pulmonary resection safely, without massive bleeding in those continuing aspirin. However, discontinuing aspirin could lead to a higher risk of an unanticipated major adverse cardiac and cerebrovascular event (MACCE). Regarding the safety of VATS, and considering the risk of MACCE causing disabling and/or fatal outcomes after lung cancer surgery, we concluded that continuing aspirin is worthwhile because there were no differences in bleeding events, thrombotic events, and operative times between the aspirin group (group 2) and nonaspirin group (group 1). Ultrasound is a cost-effective imaging modality that permits clinicians to obtain information regarding the thorax without the risk of exposure to ionizing radiation, and it provides real-time and immediate results (23). Because it is easy to use, easy to learn how to use it, portable, and accurate when examining the pleural space, it has allowed for safer pleural procedures, including VATS.

This study had two main limitations. It was a singleinstitute study with a small sample size. Therefore, it is important and necessary to perform additional investigations involving a large multi-institutional cohort to validate these findings. As mentioned, we did not research the expected port site adhesions during this study, and detecting adhesions near the apex site when using ultrasonography is difficult.

In conclusion, preoperative detection of pleural adhesions using transthoracic ultrasonography was useful for appropriately selecting pulmonary resection patients who continued using aspirin during the perioperative period.

\section{Conflicts of Interest}

The Authors declare that they have no conflict of interest in regard to this study.

\section{Authors' Contributions}

MY is the corresponding Author and wrote the manuscript. RT, NM, and TH performed the ultrasonography diagnosis. TK, NK, NS, TT, and MY participated in the surgery and postoperative management. TT, JT, NH, and ST supervised the writing of the manuscript. All Authors read and approved the final manuscript.

\section{Acknowledgements}

The Authors would like to thank Editage (www.editage.jp) for English language editing.

\section{References}

1 Passera E and Rocco G: From full thoracotomy to uniportal video-assisted thoracic surgery: lessons learned. J Vis Surg 3: 36, 2017. PMID: 29078599. DOI: 10.21037/jovs.2017.01.14

2 Chung JH, Choi YS, Cho JH, Kim HK, Kim J, Zo JI and Shim YM: Uniportal video-assisted thoracoscopic lobectomy: an alternative to conventional thoracoscopic lobectomy in lung cancer surgery? Interact Cardiovasc Thorac Surg 20: 813-819, 2015. PMID: 25736285. DOI: $10.1093 /$ icvts/ivv034

3 Pischik VG: Technical difficulties and extending the indications for VATS lobectomy. J Thorac Dis 6: S623-S630, 2014. PMID: 25379200. DOI: 10.3978/j.issn.2072-1439.2014.10.11

4 Kanzaki R, Inoue M, Minami M, Shintani Y, Nakagiri T, Funaki $\mathrm{S}$, Sawabata $\mathrm{N}$ and Okumura $\mathrm{M}$ : Feasibility of aspirin continuation during the perioperative period for pulmonary resection in lung cancer patients: a retrospective study at a single institute in Japan. Surg Today 44: 2243-2248, 2014. PMID: 24496978. DOI: 10.1007/s00595-014-0843-2

5 Sasaki M, Kawabe M, Hirai S, Yamada N, Morioka K, Ihaya A and Tanaka K: Preoperative detection of pleural adhesions by chest ultrasonography. Ann Thorac Surg 80: 439-442, 2005. PMID: 16039181. DOI: 10.1016/j.athoracsur.2005.03.021

6 Wei B, Wang T, Jiang F and Wang H: Use of transthoracic ultrasound to predict pleural adhesions: a prospective blinded study. Thorac Cardiovasc Surg 60: 101-104, 2012. PMID: 21442577. DOI: $10.1055 / \mathrm{s}-0030-1270760$

7 Cassanelli N, Caroli G, Dolci G, Dell'Amore A, Luciano G, Bini A and Stella F: Accuracy of transthoracic ultrasound for the detection of pleural adhesions. Eur J Cardiothorac Surg 42: 813-818, 2012. PMID: 22518039. DOI: 10.1093/ ejcts/ezs 144

8 Endoh H, Yamamoto R, Satoh Y, Kuwano H and Nishizawa N: Risk analysis of pulmonary resection for elderly patients with lung cancer. Surg Today 43: 514-520, 2013. PMID: 23124708. DOI: $10.1007 / \mathrm{s} 00595-012-0401-8$

9 Ansa BE, Hoffman Z, Lewis N, Savoy C, Hickson A, Stone R and Johnson T: Aspirin use among adults with cardiovascular disease in the United States: Implications for an intervention approach. J Clin Med 8: 264, 2019. PMID: 30791560. DOI: $10.3390 / \mathrm{jcm} 8020264$ 
10 Holcomb CN, Graham LA, Richman JS, Rhyne RR, Itani KM, Maddox TM and Hawn MT: The incremental risk of noncardiac surgery on adverse cardiac events following coronary stenting. J Am Coll Cardiol 64: 2730-2739, 2014. PMID: 25541124. DOI: 10.1016/j.jacc.2014.09.072

11 Mashour GA, Shanks AM and Kheterpal S: Perioperative stroke and associated mortality after noncardiac, nonneurologic surgery. Anesthesiology 114: 1289-1296, 2011. PMID: 21478735. DOI: 10.1097/ALN.0b013e318216e7f4

12 Serruys PW, Kutryk MJ and Ong AT: Coronary-artery stents. N Engl J Med 354: 483-495, 2006. PMID: 16452560. DOI: 10.1056/NEJMra051091

13 Vecenzi MN, Meislitzer T, Heitzinger B, Halaj M, Fleisher LA and Metzler $\mathrm{H}$ : Coronary artery stenting and non-cardiac surgery-a prospective outcome study. Br J Anaesth 96: 686-693, 2006. PMID: 16670113. DOI: 10.1093/bja/ael083

14 Fleisher LA, Beckman JA, Brown KA, Calkins H, Chaikof E, Fleischmann KE, Freeman WK, Froehlich JB, Kasper EK, Kersten JR, Riegel B, Robb JF, Smith SC Jr, Jacobs AK, Adams $\mathrm{CD}$, Anderson JL, Antman EM, Buller CE, Creager MA, Ettinger SM, Faxon DP, Fuster V, Halperin JL, Hiratzka JF, Hunt SA, Lytle BW, Nishimura R, Ornato JP, Page RL, Tarkington LG, Yancy $\mathrm{CW}$; American College of Cardiology/American Heart Association Task Force on Practice Guidelines (Writing Committee to Revise the 2002 Guidelines on Perioperative Cardiovascular Evaluation for Noncardiac Surgery); American Society of Echocardiography; American Society of Nuclear Cardiology; Heart Rhythm Society; Society of Cardiovascular Anesthesiologists; Society for Cardiovascular Angiography and Interventions; Society for Vascular Medicine and Biology; Society for Vascular Surgery: ACC/AHA 2007 guidelines on perioperative cardiovascular evaluation and care for noncardiac surgery: A report of the American College of Cardiology/American Heart Association Task Force on Practice Guidelines (Writing Committee to Revise the 2002 Guidelines on Perioperative Cardiovascular Evaluation for Noncardiac Surgery): developed in collaboration with the American Society of Echocardiography, American Society of Nuclear Cardiology, Heart Rhythm Society, Society of Cardiovascular Anesthesiologists, Society for Cardiovascular Angiography and Interventions, Society for Vascular Medicine and Biology, and Society for Vascular Surgery. Circulation 116: e418-e499, 2007. PMID: 17901357. DOI: 10.1161/CIRCULATIONAHA.107.185699

15 O'Riordan JM, Margey RJ, Blake G and O'Connell PR: Antiplatelet agents in the perioperative period. Arch Surg 144: 69-76, 2009. PMID: 19153328. DOI: 10.1001/archsurg.144.1.69

16 Kanda Y: Investigation of the freely available easy-to-use software 'EZR' for medical statistics. Bone Marrow Transplant 48: 452458, 2013. PMID: 23208313. DOI: 10.1038/ bmt.2012.244

17 Devereaux PJ, Mrkobrada M, Sessler DI, Leslie K, AlonsoCoello P, Kurz A, Villar JC, Sigamani A, Biccard BM, Meyhoff CS, Parlow JL, Guyatt G, Robinson A, Garg AX, Rodseth RN, Botto F, Lurati Buse G, Xavier D, Chan MT, Tiboni M, Cook D, Kumar PA, Forget P, Malaga G, Fleischmann E, Amir M,
Eikelboom J, Mizera R, Torres D, Wang CY, VanHelder T, Paniagua P, Berwanger O, Srinathan S, Graham M, Pasin L, Le Manach Y, Gao P, Pogue J, Whitlock R, Lamy A, Kearon C, Baigent C, Chow C, Pettit S, Chrolavicius S and Yusuf S; POISE-2 Investigators: Aspirin in patients undergoing noncardiac surgery. N Engl J Med 370: 1494-1503, 2014. PMID: 24679062. DOI: 10.1056/NEJMoa1401105

18 Oscarsson A, Gupta A, Fredrikson M, Järhult J, Nyström M, Darvish B, Krook H, Swahn E and Eintrei C: To continue or discontinue aspirin in the perioperative period: A randomized, controlled clinical trial. Br J Anaesth 104: 305-312, 2010. PMID: 20150346. DOI: 10.1093/bja/aeq003

19 Mantz J, Samama CM, Tubach F, Devereaux PJ, Collet JP, Albaladejo P, Cholley B, Nizard R, Barré J, Piriou V, Poirier N, Mignon A, Schlumberger S, Longrois D, Aubrun F, Farèse ME, Ravaud P and Steg PG; Stratagem Study Group: Impact of preoperative maintenance or interruption of aspirin on thrombotic and bleeding events after elective non-cardiac surgery: The multicentre, randomized, blinded, placebocontrolled, STRATAGEM trial. Br J Anaesth 107: 899-910, 2011. PMID: 21873632. DOI: 10.1093/bja/aer274

20 Schoenefeld E, Donas K, Radicke A, Osada N, Austermann M and Torsello G: Perioperative use of aspirin for patients undergoing carotid endarterectomy. Vasa 41: 282-287, 2012. PMID: 22825862. DOI: 10.1024/0301-1526/a000204

21 Vandvik PO, Lincoff AM, Gore JM, Gutterman DD, Sonnenberg FA, Alonso-Coello P, Akl EA, Lansberg MG, Guyatt GH and Spencer FA: Primary and secondary prevention of cardiovascular disease: Antithrombotic therapy and prevention of thrombosis: American College of Chest Physicians evidence-based clinical practice guidelines. Chest 141: e637S-e668S, 2012. PMID: 22315274. DOI: $10.1378 /$ chest.11-2306

22 Task Force for Preoperative Cardiav Risk Assessment and Perioperative Cardiac Management in Non-cardiac Surgery; European Society of Cardiology (ESC), Poldermans D, Bax JJ, Boerama E, De Heart S, Eeckhout E, Fowkes G, Gorenek B, Hennerici MG, Iung B, Kelm M, Kjeldsen KP, Kristensen SD, Lopez-Sendon J, Pelosi P, Philippe F, Pierard L, Ponikowski P, Schmid JP, Sellevold OF, Sicari R, Van den Berghe G and Vermassen F: Guidelines for pre-operative cardiac risk assessment and perioperative cardiac management in non-cardiac surgery. Eur Heart J 30: 2769-2812, 2009. PMID: 19713421. DOI: 10.1093/eurheartj/ehp337

23 Shojaee S and Argento AC: Ultrasound-guided pleural access. Semin Respir Crit Care Med 35: 693-705, 2014. PMID: 25463160. DOI: $10.1055 / \mathrm{s}-0034-1395794$ 